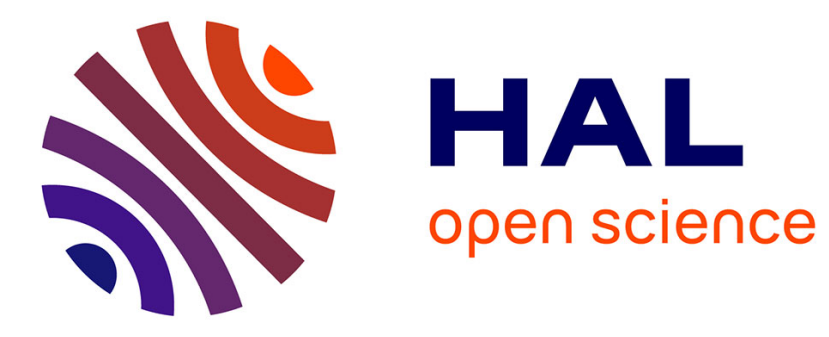

\title{
Que voit-on dans les poèmes héroïques des années 1650 ?
}

Marine Roussillon

\section{To cite this version:}

Marine Roussillon. Que voit-on dans les poèmes héroïques des années 1650 ?. Littératures classiques, 2013, L'Oeil classique, 3 (82), pp.247-259. 10.3917/licla.082.0247 . halshs-01487314

\section{HAL Id: halshs-01487314 https://shs.hal.science/halshs-01487314}

Submitted on 11 Mar 2017

HAL is a multi-disciplinary open access archive for the deposit and dissemination of scientific research documents, whether they are published or not. The documents may come from teaching and research institutions in France or abroad, or from public or private research centers.
L'archive ouverte pluridisciplinaire HAL, est destinée au dépôt et à la diffusion de documents scientifiques de niveau recherche, publiés ou non, émanant des établissements d'enseignement et de recherche français ou étrangers, des laboratoires publics ou privés. 


\section{QUE VOIT-ON DANS LES POÈMES HÉROÏQUES DES ANNÉES 1650 ? \\ Marine Roussillon}

Armand Colin | «Littératures classiques »

2013/3 N 82 | pages 247 à 259

ISSN 0992-5279

ISBN 9782200928605

Article disponible en ligne à l'adresse :

http://www.cairn.info/revue-litteratures-classiques1-2013-3-page-247.htm

\section{Pour citer cet article :}

Marine Roussillon, «Que voit-on dans les poèmes héroïques des années 1650 ? 》, Littératures classiques 2013/3 (N82), p. 247-259.

DOI 10.3917/licla.082.0247

Distribution électronique Cairn.info pour Armand Colin.

(C) Armand Colin. Tous droits réservés pour tous pays.

La reproduction ou représentation de cet article, notamment par photocopie, n'est autorisée que dans les limites des conditions générales d'utilisation du site ou, le cas échéant, des conditions générales de la licence souscrite par votre établissement. Toute autre reproduction ou représentation, en tout ou partie, sous quelque forme et de quelque manière que ce soit, est interdite sauf accord préalable et écrit de l'éditeur, en dehors des cas prévus par la législation en vigueur en France. Il est précisé que son stockage dans une base de données est également interdit. 


\section{Marine Roussillon}

\section{QUE VOIT-ON DANS LES POÈMES HÉROÏQUES DES ANNÉES 1650?}

Entre 1654 et 1658, le libraire Augustin Courbé publie quatre poèmes héroïques : Alaric ou Rome vaincue de Georges de Scudéry, La Pucelle ou la France délivrée de Jean Chapelain, Clovis ou la France chrétienne de Jean Desmarets de Saint-Sorlin et une deuxième édition du Saint Louis ou la couronne reconquise du père jésuite Le Moyne ${ }^{1}$. Ces poèmes tentent de ressusciter le genre épique tout en l'inscrivant dans un cadre national, monarchique et chrétien ${ }^{2}$. Ils partagent un même aspect matériel (des volumes de grand format illustrés) et visent un même public, rassemblant les érudits et les mondains. Les propos théoriques qui les encadrent - traités, préfaces ou avis aux lecteurs - utilisent de manière récurrente la métaphore visuelle pour décrire les textes et leurs effets, dans la continuité de la tradition de comparaison des arts ouverte par l'ut pictura poesis d'Horace. Ils définissent les poèmes non seulement comme des images merveilleuses visant l'admiration (c'est-à-dire, au sens propre, le regard étonné) mais aussi comme des portraits de leurs dédicataires. Dans le texte même des poèmes, les scènes de visions sont nombreuses, depuis les traditionnelles ekphrasis inspirées d'Homère ou de Virgile jusqu'aux visions miraculeuses propres à l'esthétique chrétienne. Enfin, tous ces poèmes sont illustrés et proposent donc de véritables images à

1. G. de Scudéry, Alaric ou Rome vaincue, Paris, Courbé, 1654 ; J. Chapelain, La Pucelle ou la France délivrée, Paris, Courbé, 1656 ; J. Desmarets de Saint-Sorlin, Clovis ou la France chrestienne, Paris, Courbé, 1657 ; P. Le Moyne, Saint Louys ou la sainte couronne reconquise, Paris, Courbé, 1658. La première édition du poème de Le Moyne a paru en 1656 chez Du Mesnil. Seul le poème de Scudéry a fait l'objet d'une édition critique : Alaric ou Rome vaincue, éd. R. Galli Pellegrini et C. Bernazzoli, Fasano / Paris, Schena / Didier Érudition, 1998. Je cite l'édition originale.

2. Sur ces poèmes, voir R. A. Sayce, The French Biblical Epic in the Seventeenth Century, Oxford, Clarendon Press, 1955 ; K. Csürös, Variétés et vicissitudes du genre épique de Ronsard à Voltaire, Paris, Champion, 1999 ; F. Wild (dir.), Épopée et mémoire nationale au XVIIe siècle, Caen, Presses Universitaires de Caen, 2011. 


\section{Marine Roussillon}

côté des descriptions ou des récits de visions. Ils produisent ainsi un dispositif complexe, dans lequel le discours sur le poème et sur sa lecture prend la forme d'un discours sur la vision, et qui associe la production d'images (textuelles ou graphiques] à un discours théorique qui en encadre la réception.

Ces poèmes participent de la construction d'une littérature moderne ${ }^{3}$, revendiquant une esthétique nationale et chrétienne et une utilité politique. L'importance qu'ils accordent à la vision s'inscrit dans cette construction. La production d'images est d'abord un moyen de rivaliser avec l'Antiquité et de montrer la valeur de l'imaginaire national et chrétien. Les descriptions d'œuvres d'art servent ainsi de support à l'affirmation de la supériorité de la langue française sur la langue latine ${ }^{4}$. L'image sert aussi à représenter le pouvoir, comme en témoigne la métaphore du portrait. Dans cette perspective, elle est pensée comme un ornement, capable d'augmenter le plaisir du lecteur-spectateur, voire d'élargir le public touché par le poème. Les illustrations rapprochent ainsi les poèmes hérö̈ques des romans édités par Courbé quelques années plus tôt, pour mieux séduire le public mondain. La promotion de la vision participe donc de la construction d'une esthétique moderne, et à travers elle d'une revendication d'utilité politique des lettres et des arts : les poèmes offrent à un large public une représentation du pouvoir à admirer et servent ainsi les intérêts de la monarchie suédoise (dans le cas d'Alaric) ou française (pour les autres poèmes). À travers l'étude de plusieurs exemples de métaphores visuelles, de récits de visions et d'images, il s'agit ici de décrire les façons dont ces poèmes pensent le lien entre une expérience esthétique de vision et une adhésion d'ordre politique.

\section{Passé et présent : une question de perspective}

«Le Temps a ses Perspectives comme le Lieu a les siennes ${ }^{5}$ » écrit le Père Le Moyne dans le Traité du poème héroïque qui sert de préface à Saint Louis. La métaphore visuelle permet de soulever la question du choix du sujet du poème et celle des relations entre le passé représenté et le présent du lecteur. Le poème héroïque doit prendre son sujet dans le passé : «l'éloignement qui détruit

3. Sur la notion de modernité et la construction d'une esthétique moderne bien avant les querelles de la fin du siècle, voir Seventeenth Century French Studies, n² 29, 2007 (« Modernités/ Modernities 》).

4. Voir D. Moncond'huy, « La galerie et l'épopée au milieu du XVII siècle », dans J.-P. Guillerm (dir.], Récits/Tableaux, Presses Universitaires de Lille, 1994, p. 103-118, en particulier p. 105-106.

5. P. Le Moyne, «Traité du poème héroïque », op. cit., n. p. 
l'apparence des choses, et les réduit au petit pied, dans les Perspectives du Lieu, les amplifie, et les augmente au double, et au triple dans les Perspectives du Temps ». La distance est ainsi nécessaire à la magnificence épique. Cependant, cette distance est susceptible de nuire au plaisir du lecteur-spectateur. Le passé, s'il est trop lointain, trop différent du présent, risque d'《 offens[er] la vue » et de « bless[er] l'imagination ${ }^{6}$ ». Au poète donc, de trouver la distance juste : une distance qui permette l'admiration sans empêcher l'adhésion. Le problème est esthétique : il s'agit de rendre possible le plaisir du lecteur en lui proposant un spectacle agréable, orné des charmes de l'exotisme mais acceptable pour son goût moderne. Le problème est aussi politique : il s'agit de montrer les rois du passé de manière vraisemblable, mais aussi comme les incarnations de valeurs encore actuelles. Pour plaire au lecteur du présent et susciter son adhésion au pouvoir en place, il s'agit de conjuguer passé et présent dans un même récit, pensé ici comme une image.

Desmarets de Saint-Sorlin déclare vouloir tirer « des ombres de l'oubli » et de l'« obscurité des temps » les «nombreux exploits de splendeur éclatants 》 de Clovis? C'est aussi l'« éclatante histoire ${ }^{8} 》$ d'Alaric que Scudéry veut donner à voir. Mais dans le même temps, il affirme que son poème peint la reine Christine de Suède, dédicataire du poème :

je sais que ce n'est pas à un broyeur d'ocre à oser entreprendre de vous peindre : mais si ma force a répondu à mon courage et à mon zèle, une belle reine Amazone aura peut-être son Apelles, comme Alexandre avait le sien. ${ }^{9}$

La métaphore de la peinture sert ici de support à un déplacement allégorique, à un changement de sujet du poème : I'histoire d'Alaric est le portrait de Christine. De la même manière, l'épître au duc de Longueville qui précède La Pucelle de Chapelain affirme que le dédicataire est le véritable modèle de la représentation offerte par le poème :

Car, à ne dissimuler rien, [les exploits de la Pucelle] ont été touchés sur l'idée de vos actions héroïques, et, pour leur donner plus de lustre, on a eu les yeux beaucoup moins sur elle que sur vous. On a mieux aimé n'être pas si précisément véritable, en les traçant sur votre modèle, que de les faire paraître moins merveilleux, en les copiant sur le sien. V.A. se reconnaîtra dans tous les projets de cette guerrière ; elle se remarquera dans tous ses combats ; elle n'y

6. Ibid., n. p.

7. J. Desmarets de Saint-Sorlin, op. cit., p. 2.

8. G. de Scudéry, op. cit., p. 5.

9. Ibid., «Épître à la sérénissime reine de Suède », n. p. 


\section{Marine Roussillon}

trouvera que les temps et les noms de changés; enfin, elle n'y verra pas tant les aventures de la Pucelle, que les siennes propres. ${ }^{10}$

La Pucelle et Alaric proposeraient ainsi des tableaux d'histoire «à clé 》 : le lecteurspectateur devrait reconnaître, derrière l'image du passé qui lui est proposée, une image du présent, comme il reconnaîtra quelques années plus tard Louis XIV dans l'Alexandre des tableaux de Le Brun. L'efficacité politique des images proposées par le texte repose donc sur un processus de reconnaissance. Reste alors à comprendre ce qui rend cette reconnaissance possible. Quelles sont les règles de cette anamorphose, qui permet de voir le visage de Christine dans le tableau d'Alaric ? Ce problème de relation entre les deux images est aussi un problème de temporalité : comment rendre visible un passé tout en en faisant une image du présent ?

\section{Tableaux d'histoire : l'image et le récit}

Les poèmes proposent de nombreuses descriptions de tableaux représentant le passé. Dans le deuxième livre du Clovis, le prince Aubéron montre au jeune roi une série de «cadres » où sont peints les exploits de ses ancêtres ${ }^{11}$. Au début de Saint Louis, ses ennemis offrent au roi des armes décorées de l'histoire de l'Égypte ${ }^{12}$. En réponse, on montre aux ambassadeurs une « superbe » tente qui représente I'histoire du règne de Louis $\mid X^{13}$. Ces ensembles d'images servent de support à des récits tout entiers orientés vers le présent : réciter le lignage de Clovis ou raconter I'histoire de Louis IX, c'est faire leur portrait ; montrer l'histoire de l'Égypte, c'est dire sa puissance. Dès lors, ces galeries de peintures et les récits qu'elles suscitent fournissent un modèle pour comprendre le récit épique ${ }^{14}$ et la manière dont il articule image du passé et représentation du pouvoir présent. Au livre IX du poème de Desmarets, la description des armes de Clovis, sur lesquelles figurent les exploits de ses descendants jusqu'à Louis XIV, assure ainsi le lien entre le roi des Francs et le roi de France, dont elle dresse le portrait par l'explicitation de son lignage. Le poème épique fonde la gloire de son dédicataire dans sa généalogie : les images merveilleuses qu'il propose reconstituent la continuité d'un lignage

10. J. Chapelain, « Épître à son altesse Monseigneur Henri d'Orléans, duc de Longueville », op. cit., n.p.
11. J. Desmarets de Saint-Sorlin, op. cit., p. 23-33.
12. P. Le Moyne, op. cit., p. 23.
13. Ibid., p. 25-31.
14. Sur ces galeries, voir D. Moncond'huy, art. cit. 
dont l'éclat rejaillit sur celui qui l'incarne présentement. Au livre VIII de Saint Louis, une voix divine propose au roi de lui « découvrir les portraits et l'histoire » de ses descendants ${ }^{15}$. Comme ces galeries historiques ou généalogiques, le poème, lui-même constitué en galerie par la mise en série des descriptions, propose des images éblouissantes du passé qui inscrivent le détenteur actuel du pouvoir dans une histoire, dans une épaisseur temporelle. La vision - celle des tableaux, de la tente ou des armes - n'est alors que le support ou le prétexte d'un récit. L'image ne se suffit pas à elle-même : elle est prise dans une série qui met en scène une succession et reconstitue la temporalité linéaire d'une narration.

Ces galeries généalogiques sont d'ailleurs le plus souvent ignorées par les illustrations. Tout se passe donc comme si elles n'offraient rien à voir, mais seulement un récit. La gravure qui ouvre le livre VII de La Pucelle est la seule à représenter l'une de ces galeries ${ }^{16}$. Elle s'inspire de l'épisode dans lequel Roger fait visiter à deux ecclésiastiques une galerie de tableaux représentant les malheurs de la France pendant la guerre de Cent Ans. Les œuvres d'art qui dans le poème servent de support au récit sont à peine esquissées dans l'image : celle-ci ne cherche pas à reproduire l'expérience de vision décrite par le texte. Elle ne construit pas non plus le spectacle de cette vision : les visages des ecclésiastiques restent dans l'ombre, tandis que seul celui de Roger, qui se détourne de la peinture pour parler à ses visiteurs, est éclairé. L'interprétation proposée par la gravure insiste donc sur la dimension avant tout narrative du passage. Le choix de la généalogie comme mode de relation entre le passé et le présent réduit la vision à un ornement, ou à une métaphore. Le poème épique n'est pas tant un portrait du roi en anamorphose qu'une succession d'images constituant par leur succession même le récit de son lignage. Les épopées des années 1650 proposeraient alors un dispositif associant d'une part le choix politique de fonder le pouvoir dans la continuité historique d'un lignage et, d'autre part, le choix esthétique de la forme narrative, particulièrement adaptée à la représentation de la succession temporelle.

Les gravures qui illustrent ces poèmes ne renoncent cependant pas pour autant à lier elles aussi passé et présent, et à revendiquer à leur manière la continuité du lignage. Le frontispice d'Alaric ${ }^{17}$ représente une statue du héros terrassant une allégorie de Rome, surmontée d'un écu aux armes de la monarchie suédoise. Les mêmes armes sont associées au monogramme de Christine de Suède dans les

15. P. Le Moyne, op. cit., p. 234.

16. http://gallica.bnf.fr/ark:/12148/bpt6k5544978m/f316.image.

17. http://gallica.bnf.fr/ark:/12148/bpt6k71340q/f2.image. 


\section{Marine Roussillon}

culs-de-lampe qui figurent à la fin de chaque livre ${ }^{18}$. On les trouve encore sur le bouclier d'Alaric dans la gravure qui ouvre le livre IX du poème ${ }^{19}$. Le blason introduit ainsi dans l'image du passé un élément du présent. Il permet la reconnaissance : Alaric est immédiatement identifié comme partageant une identité commune avec Christine. Mieux, l'insertion du blason dans l'image du passé le situe hors du temps et confère cette valeur d'éternité au lignage qu'il figure. Le blason, comme la galerie, fait du lignage l'assise de l'identité et du pouvoir du destinataire. Mais il ne figure pas la succession continue qui mène du passé au présent. II donne à voir ce qu'il y a de commun entre le passé et le présent, dans un raccourci qui nie la temporalité historique. La reconnaissance est ici au fondement d'une identité : une équivalence entre passé et présent, qui échappe à l'histoire et qui la nie. Dans les gravures qui illustrent Saint Louis et Clovis ${ }^{20}$, les fleurs de lys jouent le même rôle : elles affirment l'identité de la monarchie française entre le roi du passé et celui du présent, et du même coup, confèrent à cette monarchie la valeur de l'éternité.

Alors que les galeries et les récits dont elles sont l'objet instaurent entre passé et présent une relation de succession, et fondent ainsi le pouvoir qu'ils représentent dans sa généalogie, les gravures mettent en place entre passé et présent une relation d'identité et construisent une représentation du pouvoir qui échappe au temps. La reconnaissance, au fondement du plaisir esthétique du spectateur, est alors la condition de l'efficacité politique de l'image.

\section{La vision comme relation : l'élection divine}

Si les illustrations des poèmes épiques représentent rarement les œuvres d'art décrites dans et par le texte, il leur arrive fréquemment de choisir pour sujet une scène de vision, en particulier lorsqu'il s'agit de visions miraculeuses. L'illustration donne alors la vision en spectacle et produit une interprétation de l'acte de voir. Au livre IV du Clovis de Desmarets, Clotilde est conduite par la Vierge Marie dans un temple de cristal, «le Temple de la Vérité », dans lequel elle contemple sa propre histoire et celle de ses descendants. II s'agit d'une galerie historique semblable à celles déjà mentionnées, qui fait se succéder images du passé et images

18. Par exemple à la fin du premier livre : http://gallica.bnf.fr/ark:/12148/bpt6k71340q/f91.image.

19. http://gallica.bnf.fr/ark:/12148/bpt6k71340q/f37?.image.

20. Sur celles-ci, voir V. Meyer, « L'illustration du Saint Louis du Père Le Moyne », Cahiers de l'Association Internationale des Études Françaises, n 57, 2005, p. 47-73 ; H. G. Hall, « Three illustrated works of Desmarets de Saint-Sorlin », The Yale University Library Gazette, vol. 33, n 1, juillet 1958, p. 18-28. 
prophétiques. L'assimilation de la succession des images à un récit y est même plus explicite qu'ailleurs : Clotilde «voi[t] dans le cristal cent vérités écrites ${ }^{21}$ »et « lit [...] en lettres plus profondes ${ }^{22}$ » le baptême de Clovis. Vision et narration sont analogues, et le Temple est alors une image du poème : Clotilde $y$ lit d'ailleurs ce que le lecteur lui-même vient de lire dans le livre, ou lira quelques pages plus loin. Pourtant, la gravure qui illustre ce quatrième livre du Clovis ${ }^{23}$ ignore entièrement la galerie, comme la dimension généalogique et prophétique de la vision, pour faire avant tout de cette dernière le moment d'une relation. En bas à gauche, Clotilde évanouie est relevée par des anges. À sa droite, Marie, debout sur un nuage, semble avancer vers elle et lui prend le bras. De son autre main, elle lui désigne le temple, qui brille au loin dans le coin supérieur droit de la gravure. Clotilde ne regarde pas le temple, mais Marie. L'enjeu de la vision n'est pas réductible à ce qui est vu et décrit par le récit (les images prophétiques) : c'est la vision comme acte qui est constituée en spectacle par l'illustration, et le spectacle en acte est celui de l'alliance nouée entre la monarchie française et Dieu.

La gravure qui prend en charge la vision miraculeuse de saint Louis, au livre VIII du poème de Le Moyne, met en place un dispositif similaire (fig. 13). Elle illustre l'épisode où Louis est enlevé au Ciel sur une nuée et porté jusqu'au trône divin au milieu des chants des saints vieillards ${ }^{24}$. Dieu lui donne alors le choix entre trois couronnes : celle du Saint Empire, celle de l'Empire d'Orient et la couronne d'épines. Louis IX fait le bon choix et s'empare de la couronne d'épines. Dans le coin supérieur gauche de la gravure, Dieu, auréolé de lumière, dirige son regard vers Louis et lui tend la couronne d'épines. Plus bas, le roi lève les yeux vers Dieu et tend le bras vers la couronne. Là encore, l'échange des regards est l'occasion d'instaurer et de rendre visible une relation. La gravure ne montre pas ce que le roi aurait vu, mais donne à voir le lien privilégié entre lui et Dieu dont témoigne son expérience de vision. On distingue en effet sur le manteau de Louis un motif de fleurs de lys qui en fait la figure du roi de France : cette gravure est la seule du volume qui le désigne ainsi. C'est seulement dans le voisinage de la présence divine que Louis apparaît comme une figure atemporelle du roi de France. De la même manière, les monogrammes et les devises qui décorent la fin et le début de chaque livre associent les symboles chrétiens (la couronne d'épines) aux symboles de la monarchie française (sceptre, main de justice, fleurs de lys ou monogrammes royaux]. Ces symboles lient la

21. J. Desmarets de Saint-Sorlin, op. cit., p. 63.

22. Ibid., p. 65.

23. http://www.culture.gouv.fr/Wave/image/joconde/0493/m051202_0005722_p.jpg.

24. P. Le Moyne, op. cit., p. 227 sq. 


\section{Marine Roussillon}

permanence de la monarchie à la permanence de la religion : si la monarchie peut traverser le temps, identique à elle-même, c'est parce qu'elle est chrétienne. L'image définit ainsi les valeurs fondatrices du principe monarchique. Cette définition est spécifique aux illustrations du Saint Louis. Néanmoins, dans le Saint Louis comme dans Clovis, le spectacle de l'apparition miraculeuse manifeste la relation privilégiée entre Dieu et la monarchie française.

Au début de La Pucelle, le roi Charles et la France sont sous le coup de la colère divine. La gravure qui ouvre le premier livre du poème ${ }^{25}$ représente pourtant une scène de vision: Jeanne apparaît à Charles en costume de bergère au milieu d'une nuée lumineuse. Charles baisse les yeux et porte la main sur son cœur. Jeanne le regarde et lève un doigt vers le ciel. À l'arrière plan, les courtisans étonnés regardent la bergère. Le geste de Jeanne donne le sens de la scène : au-delà de la bergère, c'est Dieu qu'il faut voir, c'est vers lui que les regards doivent se tourner. L'apparition de Jeanne vise à restaurer une relation perdue, en rétablissant l'échange de regards entre Dieu et le roi. Dans ces apparitions miraculeuses, la vision n'est donc pas conçue comme un prétexte ou un support à la narration mais comme une mise en relation. Elle produit ainsi une tout autre représentation du pouvoir : il n'est pas légitimé par une généalogie, mais par une élection ; autrement dit, il ne résulte pas de son inscription dans un lignage, mais du mérite personnel de celui qui l'incarne.

\section{Voir, croire, désirer}

Le doigt levé de Jeanne fait signe vers Dieu, invisible mais présent. Les apparitions miraculeuses répondent ainsi à une nécessité du poème épique chrétien : rendre manifeste la présence de Dieu dans l'histoire des hommes. Cependant, la croyance que ces visions suscitent est fragile. Si elles s'imposent parfois aux héros avec la force de l'évidence, elles éveillent aussi souvent le doute. Par exemple, lorsque Alaric, qu'un ange a appelé à aller triompher de Rome, raconte ce qu'il a vu à ses conseillers, il est immédiatement averti :

Et malgré les conseils de cette vision, Craignez d'être trompé, par une illusion.

Connaissez du Démon la malice première :

Cet Ange de ténèbres, en Ange de lumière, S'est changé mille fois, pour perdre les Mortels,

Et pour leur inspirer des desseins criminels. ${ }^{26}$

25. http://gallica.bnf.fr/ark:/12148/bpt6k5544978m/f59.image.

26. G. de Scudéry, op. cit., p. 19. 
L'avertissement est d'autant plus justifié que les illusions diaboliques sont en effet fréquentes dans le poème. De la même manière, lorsque Jeanne apparaît à la cour de France, les nobles doutent d'abord de ce qu'ils voient :

Chacun plein de surprise, à ce nouveau spectacle, Doute si c'est un songe, ou si c'est un miracle $[\ldots]^{27}$

Ce qui assure alors le pouvoir de la vision, ce qui lui permet de s'imposer et d'emporter l'assentiment des spectateurs, c'est la foi. Le grand prêtre conseille à Alaric de tourner ses regards vers l'intérieur, et d'examiner sa propre foi pour juger de la vérité de sa vision:

Examinez-vous bien ; connaissez bien votre âme ;

Voyez si pour le Ciel elle est toute de flamme;

Et si pour mériter une telle faveur,

Cette âme à son devoir égale sa ferveur. ${ }^{28}$

Dans La Pucelle, Dieu intervient pour donner la foi aux spectateurs et mettre fin à leurs doutes:

En ce même moment l'auguste Providence,

Qui veut que désormais le saint Euvre commence,

Du souffle de son sein, dans leur sein descendu,

Détermine en son choix leur esprit suspendu.

Avec ce sacré souffle, une forte lumière

Leur descend dans le cœur, leur ouvre la paupière,

Et pour croire en la Fille, et recevoir sa loi,

Captive leur raison, et leur donne la foi. ${ }^{29}$

Là aussi, la vision est intérieure : c'est « le cœur » des spectateurs que la lumière divine éclaire. Dans les deux cas, la vision n'est qu'une étape vers un au-delà du visible. Elle ne montre pas, elle indique. Pour voir véritablement, il faut voir par delà ce qui est montré, et avec d'autres yeux que ceux du corps. II faut que Dieu « ouvre la paupière » du spectateur, tout comme Marie maintient ouvertes les «paupières » de Clotilde dans le Temple de la Vérité :

Clotilde entre; et ses yeux sont frappés de lumières

Que ne peuvent porter ses mortelles paupières

27. J. Chapelain, op. cit., p. 33.

28. G. de Scudéry, op. cit., p. 19.

29. J. Chapelain, op. cit., p. 33-34. 


\section{Marine Roussillon}

Mais la Reine du Ciel les touche de sa main;

Et redouble la force à son regard humain. ${ }^{30}$

Si les images peuvent être considérées comme des figures du poème, ces discours sur la vision en fournissent le protocole de lecture. Ces images éclatantes ne doivent pas être contemplées pour elles-mêmes, mais en tant qu'elles ouvrent sur un au-dehors de la vision sensible. Cette mise en scène d'une visibilité rendue possible par la foi fait écho au discours des dédicaces qui révèlent la présence d'une image cachée (le portrait du dédicataire) derrière les images du poème. Dans les deux cas, le poème nous avertit : il y a autre chose à voir que ce qu'il nous montre.

Dans les illustrations des poèmes, on observe souvent un dispositif de dédoublement, qui semble traduire cette tension du spectacle épique vers un au-delà de lui-même. Plusieurs gravures du Saint Louis citent ainsi un imaginaire chrétien et superposent dans une même image la figuration d'une scène biblique et l'illustration du récit. Ainsi, la gravure qui ouvre le livre XIII montre Lisamante assassinant le sultan, encouragée par l'apparition de Judith. Là où le texte fait parler Judith pour souligner la similitude des situations, la gravure ne permet pas d'identifier l'apparition, mais construit la scène sur le modèle du meurtre d'Holopherne, rendant la similitude visible. Ce procédé de citation picturale donne une profondeur supplémentaire à la représentation, en associant l'image du passé à une autre image, reconnaissable par le spectateur. Le dédoublement de l'image est plus visible encore dans les gravures qui illustrent le Clovis de Desmarets de Saint-Sorlin. L'illustration du récit n'occupe en effet que le tiers central de la gravure. En dessous et au-dessus de chaque vignette historiée, figurent des compositions ornementales faites de rinceaux agrémentés de symboles qui associent la scène gravée à la représentation du pouvoir ${ }^{31}$. Les $L$ entrecroisés du roi de France sont surmontés de la couronne fleurdelisée, ou mêlés aux sceptres et surmontés de la couronne impériale. Le A du cardinal de Richelieu est surmonté de la même couronne qui figure sur ses armoiries. Ces symboles ne sont pas seulement présents dans l'image : ils l'encadrent, ils la cernent comme pour en régler l'interprétation. Ils revendiquent l'actualité politique de la scène qu'ils entourent en affirmant que les images merveilleuses du Clovis doivent être rapportées au pouvoir de la monarchie française ou du cardinal de Richelieu. De la sorte, l'image visible fonctionne ici encore comme un indice : dans le jeu de ses différentes

30. J. Desmarets de Saint-Sorlin, op. cit., p. 62.

31. Voir par exemple la gravure qui ouvre le livre XVIII : http://gallica.bnf.fr/ark:/12148/ btv1b8617149k/f376.image 
composantes, elle signale qu'elle renvoie à autre chose que ce qu'elle donne à voir. Elle n'est pas présence (iconique) - ni Dieu ni Richelieu ne sont représentés mais indice d'une présence dont la vue échappe au lecteur-spectateur.

Par cette tension entre présence et absence ${ }^{32}$, la vision de l'image suscite le désir de voir plus, ou de voir à nouveau. Ainsi, Clotilde, contemplant le spectacle de ses descendants dans le Temple de la Vérité, «forme un secret désir, quoique privé d'espoir / Que le Ciel avançât le plaisir de les voir ${ }^{33}$ ». Alaric pour sa part « a l'âme toute pleine, / De l'éclatant portrait ${ }^{34} \gg$ de la reine Christine : cette image gravée dans le cœur est l'expression d'un désir quasiment amoureux. Quelques livres plus tôt, la passion d'Alaric pour la belle Amalasonthe dont il vient de se séparer est décrite dans des termes très similaires :

II retrace en lui-même une adorable Image,

Et de son bel esprit, et de son beau visage :

Mais après chaque Image, et chaque souvenir,

Lui cause une douleur, et lui coûte un soupir. ${ }^{35}$

La vision-figure prophétique, comme la vision-figure amoureuse, conjugue présence et absence, plaisir et frustration. En indiquant une présence insaisissable, elle cherche à provoquer le désir. Elle permet ainsi de représenter le pouvoir - celui du roi de France ou celui de Christine de Suède - comme un pouvoir désirable. Ces figures de souverains aimés et désirés, comme les figures de souverains élus de Dieu, fondent le pouvoir dans le mérite personnel plutôt que dans la généalogie. Mais pour définir les critères de ce mérite, elles substituent ou superposent au modèle chrétien de l'élection divine le modèle laïc et galant de la relation amoureuse. Ici, l'efficacité politique de l'image découle directement du plaisir esthétique qu'elle suscite : voir le portrait du roi avec plaisir, c'est désirer la présence et donc le pouvoir qu'il représente.

Que voit-on dans les poèmes héroïques des années 1650 ? Comment peuvent-ils dresser à la fois le tableau admirable d'un passé glorieux et le portrait de leur dédicataire ? Les galeries de peintures et les visions prophétiques décrites dans le texte des poèmes fournissent une première réponse à cette question, un premier

32. Voir à ce sujet J. Starobinski, L'EFil vivant, Paris, Gallimard, 1961.

33. J. Desmarets de Saint-Sorlin, op. cit., p. 68.

34. G. de Scudéry, op. cit., p. 235.

35. Ibid., p. 96. 


\section{Marine Roussillon}

modèle. Elles lient présent et passé dans un rapport de succession temporelle et fondent ainsi le pouvoir et la gloire du dédicataire dans l'histoire de son lignage. Pour développer cette histoire, la forme de représentation privilégiée est celle du récit ; l'image est alors un prétexte, ou un ornement.

Au contraire, la représentation visuelle conduit à rapprocher le passé et le présent en faisant abstraction du temps, en oubliant la succession des événements qui les séparent. Les gravures qui illustrent ces poèmes héroïques définissent la vision comme une relation immédiate. La mise en image des apparitions divines, ou encore l'insertion de blasons dans les gravures, annulent la temporalité historique pour inscrire les personnages hors du temps. Elles amènent le spectateur à reconnaître du présent dans le passé : Clotilde se reconnaît dans ses descendants, tout comme le spectateur des gravures reconnaît l'histoire biblique de Judith dans celle du meurtre du sultan ou le blason de Christine de Suède dans celui d'Alaric. Ce processus de reconnaissance est essentiel à l'expérience esthétique de la vision aussi bien qu'à son efficacité politique. II rend l'image visible pour le spectateur (il permet qu'elle n'offense pas la vue, pour reprendre le mot de Le Moyne) et lui permet de revendiquer des identités.

Cette mise en relation du présent et du passé dans un rapport d'immédiateté évacue la question du lignage : le pouvoir se légitime non par la continuité d'une généalogie, mais par les qualités propres du souverain, qui le rendent semblable aux héros du passé, reconnaissable et même aimable. La nature de ce mérite personnel fait débat. Dans Saint Louis, ce sont les vertus chrétiennes qui élèvent le héros en modèle du roi de France et lui permettent d'échapper au temps. Le critère du mérite est alors l'élection divine. Ailleurs, l'identité nationale est moins étroitement liée aux valeurs chrétiennes, et un choix amoureux peut tout aussi bien servir de critère. Mais quoi qu'il en soit de la définition des qualités distinctives qui rendent le souverain présent semblable aux figures héroïques du passé, partout le regard valorise le mérite personnel comme un fondement légitime de son pouvoir.

L'importance accordée à la vision dans les poèmes héroïques des années 1650 et la valorisation du mérite qu'elle met en œuvre impliquent du même coup une défense de l'utilité politique du plaisir esthétique. Le modèle de la galerie réduit la représentation du pouvoir à la répétition plus ou moins ornée du récit historique qui le légitime et qui doit d'abord sa valeur à sa véracité. Les scènes de vision proposent, elles, une autre conception de la représentation du pouvoir et de son efficacité : il s'agit de produire une image agréable du pouvoir, qui en fasse désirer la présence. La vérité de l'image importe peu. Les poèmes écartent le débat sur l'illusion et sur le risque attaché aux visions plaisantes mais mensongères : si la foi règne dans le cœur du spectateur, il ne peut être trompé. Le plaisir esthétique 
devient alors la condition même de l'adhésion : l'assentiment au pouvoir ne procède pas seulement de la croyance au récit historique qui le fonde mais aussi du plaisir suscité par la représentation, et la reconnaissance, de son mérite.

Cette promotion conjointe du regard, du mérite personnel et du plaisir esthétique fait des poèmes héroïques des années 1650 l'un des espaces où s'élabore un dispositif moderne de représentation du pouvoir, tel que se l'est approprié la monarchie dans les années 1660, avec la mise en spectacle organisée de Louis XIV en roi galant, à la fois visible, méritant et désiré.

Marine Roussillon

University of Oxford, Worcester College 